\title{
Body composition in adolescent idiopathic scoliosis
}

\author{
Manuel Ramírez · Juana Martínez-Llorens • \\ Juan Francisco Sanchez • Joan Bagó • \\ Antoni Molina $\cdot$ Joaquim Gea $\cdot$ Enric Cáceres
}

Received: 9 January 2012/Revised: 13 July 2012/ Accepted: 27 July 2012 / Published online: 12 August 2012

(C) Springer-Verlag 2012

\begin{abstract}
Study design A controlled prospective cross-sectional case study.

Objective To investigate body mass index (BMI) and corporal composition in girls with adolescent idiopathic scoliosis (AIS) and compare them with a normal population matched by sex and age.

Summary and background data There is controversy as to whether there are real anthropometric alterations in patients with AIS. Relative to the weight or the BMI, some studies find differences and other studies do not detect them. AIS and anorexia nervosa (AN) make their debut during adolescence and both may be associated with an alteration of their subjective physical perception. Some authors propose a link between AIS and AN supported both by an alteration of physical perception and lower BMI. No studies on body composition in AIS have been published.

Methods Adolescent idiopathic scoliosis patient surgery candidates during 2008 were studied. Body composition was evaluated using the bioelectrical impedance analysis (Bodystat, Isle of Man, UK). A study population of more than 5,000 patients that was published by Kyle et al. (Nutrition
\end{abstract}

M. Ramírez $(\bowtie)$ · J. F. Sanchez · A. Molina

Servei de COT, Unidad de Raquis Hospital del Mar-IMIM,

Universitat Autónoma de Barcelona (UAB),

Paseo Marítimo 25-29, 08003 Barcelona, Spain

e-mail: MRamirez@parcdesalutmar.cat

J. Martínez-Llorens · J. Gea

Servei de Pneumologia-Unitat Recerca en Muscul i Aparell

Respiratori (URMAR), Hospital del Mar-IMIM,

CIBER de Enfermedades Respiratorias, Barcelona, Spain

J. Bagó · E. Cáceres

Servei de COT, Hospital Vall d'Hebron, Universitat Autónoma

de Barcelona (UAB), Barcelona, Catalonia, Spain
17:534-541, 2001) was chosen as a control (group 1). Another control group (group 2) of healthy volunteers matched by sex and age was selected among a school age and university population in Barcelona, Spain. A variance analysis was used to analyze differences between the mean values of the control group 1, the European control group, and the AIS patient surgery candidates (Epiinfo 6.2001). Comparisons between the AIS patients and control group 2 were performed with the $T$ Student test of unpaired samples using the SPSS 15.0 (Statistical Package Social Science) software. Results Twenty-seven women with a mean age of 17.4 years. BMI was $18.9 \mathrm{~kg} / \mathrm{m}^{2}$ (SD $1.7 ; 95 \%$ CI 18.31-19.73). In the variance analysis, a significant difference between AIS and group 1 in BMI was observed (21.0 vs. $18.9, p=0.000004)$; fat-free mass (FFM $=42.6$ vs. $38.9, p=0.0000009)$ and fat mass $(\mathrm{FM}=15.6$ vs. 13.7, $p=0.03$ ). Significant differences in BMI (22.13 vs. $18.9, p=0.001 ; 95 \% \mathrm{CI}$ difference $1.85-4.60)$, fat mass index $(\mathrm{FMi}=7.17$ vs. $4.97, p=0.000 ; 95 \% \mathrm{CI}$ difference 1.36-3.05) and fat-free mass index $(\mathrm{FFMi}=14.95$ vs. $13.09, p=0.001 ; 95 \%$ CI difference 0.26-1.86) between AIS and group 2 were also seen.

Conclusion The conclusion is that there is a real alteration of body composition in AIS. The BMI, FFMi and FMi are lower than in the general population in the series under study.

Keywords Adolescent idiopathic scoliosis - Body mass index $\cdot$ Body composition

\section{Introduction}

There is controversy as to whether there are real anthropometric alterations in patients with adolescent idiopathic 
scoliosis (AIS) [1-6]. Numerous growth studies have reported that AIS patients are taller than healthy controls [7-9]. Nevertheless, other studies did not find any difference $[10,11]$. Some authors have even reported that girls affected by scoliosis are shorter than controls [12]. A similar controversy exists with respect to weight or the body mass index (BMI). Such being that some studies find differences and others do not detect them [1, 4-6, 12-14]

Anorexia nervosa (AN) and AIS make their debut during adolescence and both may be associated with an alteration of subjective physical perception. This aspect combined with the demonstration, in some studies, of a lower BMI in patients with AIS [1, 4, 14] has led certain authors to propose a possible link between AIS and AN [4, 15]. Little has been published that shows the corporal composition of patients with AIS [12, 16, 17].

The aim of the work presented was to analyze the BMI and the corporal composition of patients with AIS in order to determine if patients with AIS suffer an anthropometric alteration.

\section{Materials}

This is a controlled prospective case-control study conducted in accordance with the World Medical Association Guidelines for Research on Humans [18]. Our Institutional Ethics Committee approved the study and all patients and controls gave written fully informed consent.

The inclusion criteria were: AIS programmed for intervention in the spine units of the Hospital del Mar and the Hospital of Valle Hebrón (Barcelona).

The exclusion criteria were patients with a previous cardiorespiratory pathology and those that refused to sign the consent form.

Corrected body height was calculated on the basis of an arm span measurement. Arm span was measured by using a wall-mounted tape measure with the subject's arms fully extended horizontally and parallel to the tape measure. Readings were taken to the nearest $0.1 \mathrm{~cm}$. Body weight was assessed using a conventional weighing chair (DRY, Berlin, Germany). BMI was calculated as the body weight divided by the square of the calculated height $\left(\mathrm{kg} / \mathrm{m}^{2}\right)$. Body composition was evaluated using bioelectrical impedance analysis (BIA) (Bodystat, Isle of Man, UK). Fat-free mass (FFM), Fat mass (FM), the percentage of fat mass (\% FM) and the percentage of water was calculated. The fat-free mass index and fat mass index (FFMi and FMi) were calculated as FFM or FM divided by the square of the height.

As a control group, a population study of more than 5,000 patients published by Kyle et al. [16] (group 1) was chosen. It was proposed as a reference to evaluate body composition in a western European population. It establishes reference values for FFM, fat mass (FM) and percentage of fat mass (\% FM) in a large white (western European) population $(n=5,225)$ grouped by age and sex. Kyle et al. calculated body composition using a BIA and data were presented in percentiles depending on sex and age [16].

In order to avoid any bias and to compare the FFMi and FMi, a second control group (group 2) was selected. It consisted of healthy volunteers matched by age and gender. They were selected through a call for volunteers among a school age and university population in Barcelona, Spain. In order to avoid selection of volunteers with scoliosis all volunteers who presented a malformation based on the ADAMS test were excluded. It permitted a comparison of FM and FFM values in accordance with height.

Values are expressed as a mean with a $95 \%$ confidence interval (CI). Since the distribution of different quantitative variables was normal (Kolmogoroff-Smirnoff test), comparisons between AIS surgery candidates and control group 2 were performed using the parametric $t$ tests of unpaired samples (SPSS 15.0, Statistical Package Social Science, Chicago). An analysis of variance was used to analyze the differences between the mean values of the group under study and the European control group [Epiinfo 6.2001, Center for Disease Control and prevention (CDC)]. A $p$ value of $\leq 0.05$ was considered statistically significant.

\section{Results}

There were 27 women with a mean age of 17.4 years (13-26). The mean Cobb angle [19] was $66^{\circ}$ (SD 15) and $35^{\circ}\left(\mathrm{SD} 16^{\circ}\right)$ for the dorsal and lumbar spine. The anthropometric and descriptive data are shown in Table 1. The mean BMI was $18.9 \mathrm{~kg} / \mathrm{m}^{2}$ (SD $1.7 ; 95 \%$ CI 17.31-19.73) it was within the normal limits, but what stands out is the percentage of (26\%) of patients with a BMI within the scoring for anorexia nervosa (AN) $\left(<17.5 \mathrm{~kg} / \mathrm{m}^{2}\right)$ and up to $55.6 \%$ scoring a low weight or undernourishment degree $1\left(<18.5 \mathrm{~kg} / \mathrm{m}^{2}\right)$ (Table 2).

Control group 1 and control group 2 are comparable (Table 3), no statistically significant differences were found, except for a slight increase of fat mass (FM $\mathrm{kg}$ ) in control group 2.

The analysis of the variance between the AIS group and control group 1 (Table 4) demonstrates statistically significant differences in terms of weight and BMI but not in terms of height. Corporal composition also demonstrates significant differences with a decrease of FFM and FM in the AIS group, but not in the percentage of FM.

When the patients with AIS were compared with control group 2, significant differences were also found in BMI 
Table 1 Descriptive dates

\begin{tabular}{lcccccr}
\hline & $N$ & Min & Max & Mean & SD & Variance \\
\hline Age & 27 & 13 & 26 & 17.44 & 3.401 & 11.564 \\
Weight & 27 & 44 & 68 & 52.85 & 6.075 & 36.900 \\
Height & 27 & 1.57 & 1.80 & 1.6700 & 0.05704 & 0.003 \\
BMI & 27 & 15.90 & 22.80 & 18.9515 & 1.76020 & 3.098 \\
FFMi & 27 & 12.5 & 15.7 & 13.993 & 1.0532 & 1.109 \\
FMi & 27 & 2.69 & 7.72 & 4.9090 & 1.25511 & 1.575 \\
$\%$ FFM & 27 & 36.30 & 85.40 & 71.8304 & 11.05532 & 122.220 \\
$\%$ FM & 27 & 14.6 & 37.7 & 25.663 & 4.8623 & 23.642 \\
FM (kg) & 27 & 7.6 & 23.9 & 13.763 & 3.8787 & 15.044 \\
$\%$ Water & 27 & 49,5 & 68.7 & 59.759 & 4.2366 & 17.949 \\
Water & 27 & 27.9 & 38.4 & 31.426 & 2.4124 & 5.820 \\
$N$ & 27 & & & & & \\
\hline
\end{tabular}

$B M I$ body mass index, $F F M i$ fat-free mass index, $F M i$ fat mass index, $\%$ FFM percentage of fat-free mass in total weight, $\% F M$ percentage of fat mass in total weight, $F M$ fat mass in kilograms

Table 2 IBM distribution and percentiles

\begin{tabular}{ll}
\hline$N$ & \\
Valid & 27 \\
Loss & 0 \\
Mean & 18.9515 \\
SD & 1.76020 \\
Variance & 3.098 \\
Percentiles & \\
25 & 17.5000 \\
50 & 18.4000 \\
75 & 20.4000 \\
\hline
\end{tabular}

(22.13 vs. $18.9, p=0.001 ; 95 \%$ CI of difference $1.85-4.60)$, weight (59.55 vs. $52.85, p=0.004 ; 95 \%$ CI of differences $3.12-10.62)$ and the FMi (7.17 vs. 4.97, $p=0.000 ; 95 \% \mathrm{CI}$ of difference 1.36-3.05) and FFMi (14.95 vs. $13.09, p=0.001 ; 95 \% \mathrm{CI}$ of difference 0.26-1.86) (Table 5).

We also found differences in the FM\% (32.12 vs. 26.07, $p=000 ; 95 \%$ CI of difference 3.30-8.78) between the AIS group and control group 2 .

\section{Discussion}

Bioelectrical impedance analysis was used to measure body composition because other available methods are expensive, time-consuming and need trained personnel. BIA is an easy and economical method that has been shown to be more accurate for determining leanness or fatness in humans [20, 21]. BIA is also validated in adolescent and young people [21].
Table 3 Analysis of variance (Epiinfo 6.2001. Centers for Disease Control and Prevention (CDC), Atlanta USA and World Health Organization Geneva, Switzerland)

\begin{tabular}{lllcl}
\hline & Mean & Variance & Sample size & $P$ \\
\hline BMI & & & & \\
Control group 1 & 21.0 & 5.29 & 488 & 0.17 \\
Control group 2 & 22.13 & 8.21 & 25 & \\
Weight & & & & \\
Control group 1 & 58.3 & 54.76 & 488 & 0.39 \\
Control group 2 & 59.55 & 46.24 & 25 & \\
Height & & & & \\
Control group 1 & 166.6 & 38.44 & 488 & 0.11 \\
Control group 2 & 167.3 & 0.0007 & 25 & \\
FFM (kg) & & & & \\
Control group 1 & 42.6 & 16.81 & 488 & \\
Control group 2 & 41.96 & 35.24 & 25 & \\
FM (kg) & & & & \\
Control group 1 & 15.6 & 20.25 & 488 & \\
Control group 2 & 17.06 & 15.04 & 25 & \\
FM (\%) & & & & \\
Control group 1 & 26.4 & 23.04 & 488 & \\
Control group 2 & 28.66 & 23.64 & 25 &
\end{tabular}

Comparison between both control groups. The both groups were comparable except for a slight difference in FM. Similar data in Mediterranean population were published by others [22]

It is not confirmed that patients with AIS display an alteration in their anthropomorphic development with an average BMI within normal values. However, the percentage of cases below undernourishment degree 1 or even within the margins of AN [4-6, 12, 14-17] is above the general population.

At the same time, they present an alteration of corporal composition. The study of the variance of the European group control demonstrates a lower BMI. It is basically due to the FFM and a lower FM is also presented. However, there was a percentage of FM without differences. We used ANOVA to compare mean values in patients group with control group 1 because the data published by Kyle have mean values, sample size and variance, but not all particular data of each case, making impossible to use unpaired $t$ Student,

Compared with control group 2, the differences are as much shown in the FMi as in FFMi and FM\%. It might be explained with the extracted data of some works that show a tendency to a greater percentage of FM in the Hispanic population [22].

Two control groups were chosen. A first group was the series published by Kyle et al. [20]. The reason being that it establishes reference values for FFM, FM and \% FM in a large white (western European) population $(n=5,225)$ grouped by age and gender. 
Table 4 Analysis of variance [Epiinfo 6.2001. Centers for Disease Control and Prevention (CDC), Atlanta USA and World Health Organization Geneva, Switzerland]

\begin{tabular}{lcccc}
\hline & Mean & Variance & Sample size & $P$ \\
\hline BMI & & & & \\
Control group & 21.0 & 5.29 & 488 & 0.000004 \\
AIS & 18.9 & 3.0 & 27 & \\
Weight & & & & \\
Control group & 58.3 & 54.76 & 488 & 0.00019 \\
AIS & 52.85 & 36.90 & 27 & \\
Height & & & & \\
Control group & 166.6 & 38.44 & 488 & \\
AIS & 167 & 0.003 & 27 & 0.000009 \\
FFM (kg) & & & & \\
Control group & 42.6 & 16.81 & 488 & \\
AIS & 38.97 & 14.92 & 27 & 0.03 \\
FM (kg) & & & & \\
Control group & 15.6 & 20.25 & 488 & \\
AIS & 13.7 & 15.04 & 27 & \\
FM (\%) & & & & \\
Control group & 26.4 & 23.04 & 488 & \\
AIS & 25.66 & 23.64 & 27 & \\
\hline
\end{tabular}

Table $5 T$ student analysis of unpaired samples between AIS patients and control group 2

\begin{tabular}{|c|c|c|c|c|c|c|c|c|}
\hline & \multicolumn{2}{|l|}{ Weight } & \multicolumn{2}{|l|}{ BMI } & \multicolumn{2}{|l|}{ FMi } & \multicolumn{2}{|l|}{ FFMi } \\
\hline & Mean & SD & Mean & SD & Mean & SD & Mean & SD \\
\hline Control 2 & 59.55 & 6.80 & 22.13 & 2.98 & 7.17 & 1.63 & 14.95 & 1.59 \\
\hline AIS & 52.85 & 6.07 & 18.9 & 1.76 & 4.97 & 1.12 & 13.09 & 1.05 \\
\hline$P$ & 0.004 & & 0.001 & & 0.000 & & 0.001 & \\
\hline
\end{tabular}

Statistically significant differences in FFMi and Fmi (SPSS 15.0, Statistical Package Social Science, Chicago)

At the same time, in order to avoid a population bias and considering that regional differences between control group 1 and 2 cannot be ascertained, a control group matched by age and gender (in order to have similar sexual maturity) permitted a comparison of FMi and FFMi.

The study of variance between the control group 1 and control group 2 shows no differences in height, weight, BMI and FFM. These data gives strength to the results obtained and therefore reaffirms the differences found.

The only difference between the control groups is a slight increase in fat mass in the control group 2 (Spanish) that would correspond with published data of Casas et al. [22]. Casas et al. published a slight increase in fat mass FM in Mediterranean population matched by age and sex. The fact that FM is the value decreased in patients with scoliosis is reaffirmed yet more with these data.
Anorexia nervosa (AN) and AIS make their debut during the adolescence and both can be associated with an alteration of subjective physical perception. This aspect combined with the demonstration, in some studies, of a smaller BMI in patients with AIS $[1,4,14]$ has led certain authors to propose a possible link between AIS and AN [15].

If we take the definition of the World Health Organization (WHO) [23], a good nutritional state is equal to a BMI ranging from 20 to $25 \mathrm{~kg} / \mathrm{m}^{2}$ (in women $20-23.9 \mathrm{~kg} /$ $\mathrm{m}^{2}$ ). A BMI of $18.5-20 \mathrm{~kg} / \mathrm{m}^{2}$ is defined as low weight and a BMI of $17-18.5 \mathrm{~kg} / \mathrm{m}^{2}$ as first degree nutritional deficiency (caloric-protein undernourishment). It is important to point out the high percentage of undernourished and low weight patients in the AIS group (55.6\% under $18.5 \mathrm{~kg}$ / $\mathrm{m}^{2}$ )

The corporal composition in AIS and AN is similar, with a weight and BMI reduction based fundamentally on the FM. FFMi was also significantly lower but to a lesser degree [24-26].

There is a well-established relationship between osteoporosis and eating disorders. This relationship has also been described in AIS [5, 27-30]. Studies of AN demonstrate that BMI and disease duration is a strong predictor of an alteration in bone turnover. Bone turnover has been shown to be markedly disturbed when the BMI falls below $15 \mathrm{~kg} / \mathrm{m}^{2}$ [5, 27-29]. Values of BMI in AIS could be also a predictor of osteoporosis.

In our series, AIS patients are more slender than their age-matched group. AIS patients have less FM. If FM reflects energy balance and is linked to endocrine function, FM may have implications in terms of growth and/or bone mass in AIS [12].

The high incidence of low body-weight, including undernourishment degree 1 in this series and other studies published [1, 4, 6, 12-15], is a cause for concern. These findings may be an unrecognized symptom of AIS [12].

An association between AIS and an eating disorder or even delimiting a group with a worse prognosis is a possibility that should be studied. Eating disorders have implications in terms of psychological well-being and an alteration of the subjective physical perception that might diminish the possibilities a satisfactory final outcome. Koch et al. [13] show that patients in a group dissatisfied with surgery results have significantly lower BMI than those in the satisfied group. Nevertheless, without analyzing the correlation between BMI and satisfaction, they concluded that psychological factors (self-image, emotional stability) are related to the satisfaction of patients.

This work confirms the existence of an altered body composition in patients with AIS, which is explained in the multifactorial origin of the AIS.

The next step would be confirm whether these patients with low weight and body composition alteration is 
associated with impaired physical perception (similar to that present in the AN) or even has an impact on the patient perceived outcome or the satisfaction with surgery outcomes.

\section{Limitations}

Considering that the $N$ in our work is very low, the differences are still statistically significant. Furthermore, to our knowledge, there are no other published works that study the alteration of body composition in AIS patients programmed for surgery. Other studies that confirm this hypothesis are needed.

\section{Conclusions}

It seems evident that patients with AIS present an alteration of their BMI.

Lowering of the BMI is associated with an alteration of corporal composition, especially at the expense of the FM. A nutritional alteration in the AIS patient may exist.

If this decrease in the BMI is correlated, as in $\mathrm{AN}$, with the osteoporosis presented in these patients other studies would be needed.

Our opinion is that, there may be an association between AIS and AN that explains some symptomatology and denotes a group with a worse surgical prognosis. It must be the subject of subsequent studies.

\section{Conflict of interest None.}

\section{References}

1. Siu King Cheung C, Tak Keung Lee W, Kit Tse Y, Ping Tang S, Man Lee K, Guo X, Qin L, Chun Yiu Cheng J (2003) Abnormal peripuberal anthropometric measurements and growth pattern in adolescent idiopathic scoliosis: a study of 598 patients. Spine 28(18):2152-2157

2. Loncar-Dusek M, Pecina M, Prebeg Z (1991) A longitudinal study of growth velocity and development of secondary gender characteristics versus onset of idiopathic scoliosis. Clin Orthop 270:278-282

3. Lonstein JE, Carlson JM (1984) The prediction of curve progression in untreated idiopathic scoliosis during growth. J Bone Joint Surg 66A:1061

4. Wei-Jun W, Xu S, Zhi-Wei W, Xu-Sheng Q, Zhen L, Yong Q (2012) Abnormal anthropometric measurements and growth pattern in male adolescent idiopathic scoliosis. Eur Spine J 21(1):77-83

5. Sadat-Ali M, Al-Othman A, Bubshait D, Al-Dakhneel D (2008) Does scoliosis causes low bone mass? A comparative study between siblings. Eur Spine J 17(7):944-947

6. Park WW, Suh KT, Kim SJ, Lee JS (2009) Decreased ostegenic differentiation of mesenchymal stem cell and reduced bone mineral density in patients with adolescent idiopathic scoliosis. Eur Spine J 18(12):1920-1926
7. Carr AJ, Jefferson RJ, Weisz I (1989) Correction of body height in scoliosis patients using ISIS scanning. Spine 14:220-222

8. Cheng JCY, Leung SSF, Chiu BSK et al (1998) Can we predict body height from segmental bone length measurements? A study of 3647 children. J Pediatr Orthop 18(3):387-393

9. Willner S (1975) The proportion of legs to trunk in girls with idiopathic structural scoliosis. Acta Orthop Scand 46:84-89

10. Drummond DS, Rogala EJ (1980) Growth and maturation of adolescents with idiopathic scoliosis. Spine 5:507-511

11. Duval-Beaupere G (1971) In: Zorab PA (ed) Scoliosis and growth. Churchill Livingstone, London, pp 58-61

12. Dangerfield PH, Davey RC, Chockalingam N, Cochrane T, Dorgan JC (2005) Body composition in females with adolescent idiopathic scoliosis. J Bone Joint Surg 88-B(Suppl II):230-231

13. Koch KD, Buchanan R, Birch JG, Morton AA, Gatchel RJ, Browne RH (2001) Adolescents undergoing surgery idiopathic scoliosis. How physical and psychological characteristics relate to patient satisfaction with the cosmetic result. Spine 26(19): $2119-2124$

14. Liu Z, Qiu Y, Qiu X, Sun X (2009) Body mass index in Chinese girls with adolescent idiopathic scoliosis. Eur Spine J 18(suppl 4):S445-S470

15. Smith FM, Latchford G, Hall RM, Millner PA, Dickson RA (2002) Indications of disordered eating behavior in adolescent patients with idiopathic scoliosis. J Bone Joint Surg 84-B: 392-394

16. Li W-G, Qiu Y (2008) Decreased lean mass in adolescent idiopathic scoliosis. Stud Health Technol Inform 140:344

17. Melendo E, Ramírez M, Martínez J, Bagó J, Saló G, Cáceres E (2007) Body composition alterations in patients with adolescent idiopathic scoliosis. Relationship with work capacity. Eur Spine J 16:137

18. The World Medical Association's Declaration of Helsinki (1964) Ethical principles for medical research involving human subjects. http://www.wma.net/s/policy/17-c_s.html

19. Cobb JR (1948) Outline for the study of scoliosis. In: American academy of orthopaedic surgeons: instructional course lectures, vol 5, pp 61-75

20. Kyle UG, Genton L, Slosman DO, Pichard C (2001) Fat free mass and fat mass percentiles in 5225 healthy subjects aged 15 to 98 years. Nutrition 17:534-541

21. Pietrobelli A et al (2003) Predicting fat free mass in children using bioimpedance analysis. Acta diabetol 40:S212-S215

22. Casas YG, Schiller BC, DeSouza CA, Seals DR (2001) Total and regional body composition across ageing in healthy Hispanic and white women of similar socioeconomic status. Am J Clin Nutr 73:13-18

23. World Health Organization (1995) The use and interpretation of antropometry. WHO, Geneva

24. Hsu LK (1996) Epidemiology of the eating disorders. Psychiatr Clin North Am 19:681-700

25. Mazess RB, Barden HS, Ohlrich ES (1990) Skeletal and bodycomposition effects of anorexia nervosa. Am J Clin Nutr $52: 438-441$

26. Garcia MT, Muñoz Calvo MT, Barrios V, Martinez G, Martos Moreno GA, Hawkins F, Argente J (2007) Regional fat distribution in adolescents with anorexia nervosa: effect of duration of malnutrition and weight recovery. Eur J Endocrinol 157:473-479

27. Weinbrenner T, Zittrmann A, Gouni-Berthold I, Stehle P, Berthold HK (2003) Body mass index and disease duration are predictors of disturbed bone turnover in anorexia nervosa. A casecontrol study. Eur J Clin Nutr 57:1262-1267

28. Zipfel S, Zipfel S, Seibel MJ, Lone B, Beaumont PK, Kasperk C, Herzg W (2001) Osteoporosis in eating disorders: a follow-up study of patients with anorexia nervosa and bulimia nervosa. J Clin Endocrinol Metab 86:5227-5233 
29. Lee WTK, Cheung CSK, Tse YK, Guo X, Qin L, Ho S, Lau J, Cheng JCY (2005) Generalized low bone mass of girls with adolescent idiopathic scoliosis is related to inadequate calcium intake and weight bearing physical activity in peripubertal period. Osteoporos Int 16:1024-1035
30. Cheng JCY, Guo X, Sher AH (1999) A persistent osteopenia in adolescent idiopathic scoliosis: a longitudinal study. Spine 24(12): 1218 